\title{
Compression of Dynamic 3D Meshes and Progressive Displaying
}

\author{
Bin-Shyan JONG $^{\dagger}$, Chi-Kang KAO ${ }^{\dagger \dagger a)}$, Nonmembers, Juin-Ling TSENG ${ }^{\dagger \dagger \dagger}$, Member, $^{\circ}$ \\ and Tsong-Wuu LIN ${ }^{\dagger \dagger \dagger}$, Nonmember
}

\begin{abstract}
SUMMARY This paper introduces a new dynamic 3D mesh representation that provides 3D animation support of progressive display and drastically reduces the amount of storage space required for $3 \mathrm{D}$ animation. The primary purpose of progressive display is to allow viewers to get animation as quickly as possible, rather than having to wait until all data has been downloaded. In other words, this method allows for the simultaneous transmission and playing of 3D animation. Experiments show that coarser 3D animation could be reconstructed with as little as $150 \mathrm{~KB}$ of data transferred. Using the sustained transmission of refined operators, viewers feel that resolution approaches that of the original animation. The methods used in this study are based on a compression technique commonly used in 3D animation - clustered principle component analysis, using the linearly independent rules of principle components, so that animation can be stored using smaller amounts of data. This method can be coupled with streaming technology to reconstruct animation through iterative updating. Each principle component is a portion of the streaming data to be stored and transmitted after compression, as well as a refined operator during the animation update process. This paper considers errors and rate-distortion optimization, and introduces weighted progressive transmitting (WPT), using refined sequences from optimized principle components, so that each refinement yields an increase in quality. In other words, with identical data size, this method allows each principle component to reduce allowable error and provide the highest quality $3 \mathrm{D}$ animation.

key words: clustered principal component analysis, dynamic $3 D$ mesh compression, progressive $3 D$ animation, progressive display of dynamic $3 D$ meshes
\end{abstract}

\section{Introduction}

3D animation can be viewed as multiple 3D static meshes being displayed sequentially, under the assumption that in a typical scene, the connectivity of models does not change from one frame to the next. For this reason, traditional method of expressing 3D animation require only one connectivity, as well as the coordinates of those vertices in models at for every frame. To avoid excessive popping while rendering the animated model, vertex displacement of neighboring frames does not significantly change. Such an approach causes repeated (identical) or similar values to

\footnotetext{
Manuscript received July 16, 2010.

Manuscript revised June 15, 2011.

${ }^{\dagger}$ The author is with the Dep. of Information and Computer Engineering, Chung Yuan Christian University, Taiwan.

${ }^{\dagger}$ The author is with the Dep. of Department of Electronic Engineering, Chung Yuan Christian University, Taiwan.

${ }^{\dagger \dagger}$ The author is with the Dep. of Computer Science and Information Engineering, Minghsin University of Science and Technology, Taiwan.

${ }^{\dagger+\dagger}$ The author is with the Dep. of Computer and Information Science, Soochow University, Taiwan.

a) E-mail: windkey@cg.ice.cycu.edu.tw

DOI: $10.1587 /$ transinf.E94.D.2271
}

exist in the geometric information of 3D animation. This means that traditional 3D animation has room for compression, and as a result, researchers have introduced compression methods for 3D animation [1]-[3].

Principal component analysis (PCA) is a just one group of compression methods. PCA converts the geometric information of 3D animation into a linear combination of eigenvectors for expression. These eigenvectors are referred to as principal components (PCs). PCA reduces data by means of dimensional reduction: after the original animation has been converted to PCs, only the first few important components are stored. The remaining PCs can be used to refine the reconstructed animation. High compression rates and applicability with any 3D animation are two of the many advantages of PCA. In fact, many other compression methods are limited to processing only manifold meshes [3], [4].

Compression methods based on PCA emphasized error reduction in the reconstruction of animation [5], [6], or the performance of second predictions to compress PCs [7]-[9]. Aside from reducing errors, the primary objective of this study was to introduce a new expressional method, for 3D animation to be transmitted and rendered progressively, as with 2D progressive images (see Fig. 1). With the sequential transmission of PCs, coarse 3D animation could be refined clearly in real-time. In practical applications, viewers could view a preview of the entire animation with a minimum of delay, only deciding whether or not they are interested in the animation after this initial viewing. They could then decide whether to cancel the browsing or continue downloading the remainder of the animation in full-resolution. This approach would allow for more effective use of internet bandwidth and increase user interaction.

Clustered PCA [5] has a better compression rate than global (non-clustering) PCA [2], but inconsistencies in the degree of change between clusters can lead to noncontinuity boundaries between clusters [10], [11]. To overcome this problem, this study optimizes each cluster of principal components. Clusters with a greater degree of change were given more PCs, while clusters with less change were given fewer PCs. In this manner we are able to balance errors between clusters. Due to similarities between errors from one cluster to the next, non-continuity surfaces could be smoothed and improved, thereby significantly reducing error overall.

The principal contributions of this paper are as follows: (1) construction of progressive 3D animation, in that the de- 


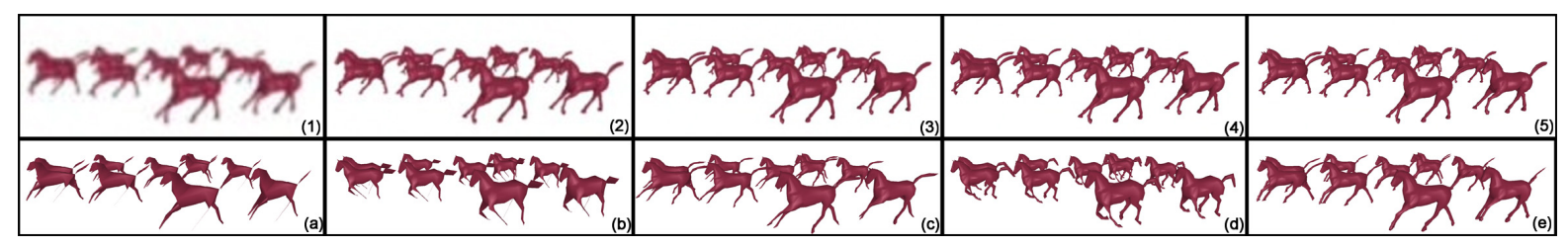

Fig. 1 The images in the first row (labeled $1 \sim 5$ ) depict of a progression of JPEG images gradually increasing in image quality, as the amount of data increases. The method proposed in this study (labeled $\mathrm{a} \sim \mathrm{e}$ ) produces progressive 3D animation, wherein the frames shown for each image are not identical, having different resolutions; in this way the viewer can freely change the viewpoint around the scene.

coding side would not have to wait until all compressed data had been received before displaying could begin; (2) optimization of PC updating sequences would reduce difficulties in determining the number of PCs, and improve the problem of non-continuity boundaries between clusters; (3) compared with PCA based compression methods, this method adds PC transmission sequences for the required storage, adding somewhat to the overhead; (4) the accelerated decoding side would be able to support real-time rendering, while the time spent decompressing would consist of transmitting/receiving PCs through networks.

\section{Related Work}

To reduce the vast required storage involved in 3D animation, Lengyel [1], Collins [12], Mamou [11], and James [13] utilized clustered prediction for compression. Their methods used the calculated affine matrix (between individual frames and a reference frame), to predict the entire animation. The residual prediction was then compressed using discrete cosine transform (DCT) or quantization. This category of methods leads to improved results for near rigid animation, but produces a great number of errors at boundaries between clusters; unsmooth surfaces must be allotted additional post-processing [11].

Ibarria [14] introduced a prediction method called Dynapack, suggesting that there were a high association of connectivity between coordinates in neighboring time and space, so it was possible to include these relationships into the predictions. Briceño [3] converted the frames (meshes) of animations into individual images using geometric images [15], and termed the method geometry video. Parameterization methods project 3D models over images, allowing for the use of any image processing technique in processing 3D animation. The errors produced in compression using geometry video are largely determined by the results of parameterization; at the same time, parameterization is limited to processing only manifold mesh animations. Guskov [4] constructed a mapping from each frame to wavelet coefficients and successfully achieved a high compression ratio if the frames were well predicted by the parameterization.

Huang [16] introduced a method based on key frames, in which the entire 3D animation was expressed with linear combinations of key frames. Lee [17] used genetic algorithm to find optimized key frames. To reduce errors in reconstruction, the changes in the edges of animation over time and the geometric error on model exteriors were used to measure whether frames could be used as key frames. The methods introduced by Huang and Lee are based on key frames, so beyond their advantages of compression, the methods were also able to produce summaries of animated content for further application. Unfortunately, these methods require some improvement in their compression ratio.

Performing compression of 3D animation using PCA was first introduced by Alexa [2], who translated geometric information into matrix form and performed PCA to divide the whole matrix into principal components. In this way, he was able to reduce the amount of data needed to store 3D animation by dimension reduction. Alexa's method performs PCA directly, for the entire 3D animation, and is therefore termed global PCA. Sattler [5] combined vertices with similar trajectories into single clusters before performing PCA for each cluster. Sattler's method was able to express vertex coordinates with fewer PCs and greater precision than global PCA. Amjoun [6] elaborated on Sattler's [5] method of introducing local coordinate frames to perform compression using PCA, after converting global coordinates into local coordinates, thereby lowering reconstruction error and improving prediction results.

A number of researchers have focused on improving the compression rate of PCA. Karni [7] suggested that, even for differing PCs, there remained a degree of connectivity with regard to time. He suggested the use of linear prediction coding (LPC), by which the first few PCs would predict later ones, thereby reducing the amount of data required. Karni's method yielded improved animation effects with more frames. In a deeper study on PCs, Vasa introduced a series of prediction functions [8], [9], [18], combining Edgebreaker [19] and parallelogram predictors to predict the PCA coefficients of neighboring vertices. To reduce errors caused by imprecision in parallelogram prediction, Vasa [9] incorporated simplified, averaged predictors similar to those introduced by Alliez [20], in the prediction of PCA coefficients of neighboring vertices, with results superior to those of parallelogram predictors. Since PCA coefficients are portions of space, Vasa's method [8] introduced a non-least square prediction method to predict PCs at different vertices in time, expressing the predicted PCA basis more precisely than Karni's method [7]. 


\section{Representing Progressive 3D Animation}

PCA is broadly applied to processing different types of data. In addition to the compression of dynamic 3D meshes, researchers have produced good results with the use of PCA in the compression of motion capture data [21], [22]. However, existing methods cannot perform reconstruction until all reserved PCs have been transmitted; lacking detailed discussion or explanation regarding how to achieve progressive updating. In the method published by Sattler [5], the number of PCs in each cluster was identical not having performed adjustments based on the degree of change in each cluster, which led to non-continuity surfaces at the boundaries of different clusters. This paper introduced a complete framework to resolve these problems described above.

\subsection{Compression of Dynamic 3D Meshes}

To reduce the amount of data needed for decompression, it is necessary to select a compression method with an adequate compression ratio. Sattler's method is efficient [5], but takes into account reconstruction error to minimize the clustering error. In other words, after each clustering stage, the method performs PCA compression, PCA decompression, and calculates the clustering error, comparing the reconstructed animation with the original animation. If the clustering stage had not converged or the initial seed selection had been inappropriate, the clustering stage would take very long time.

Our method differs from Sattler's method by dividing clustering and compression, with the common but effective K-mean clustering [23]. After performing multiple experiments, the Lloyd's algorithm provided by [23] have better results and is selected as our clustering function. In Lloyd's algorithm, the initial seed points are randomly selected. However, this method [23] performs the whole clustering for multiple times (about 10) to avoid selecting the bad seed points as results. After clustering, we compress each cluster individually by PCA. After a 3D animation mesh is clustered, each cluster has its own geometric coordinate matrix $\mathrm{A}$, where matrix $\mathrm{A}$ includes all vertex coordinate information for the entire 3D animation. The dimension of A is $M \times N$, where $\mathrm{M}$ is the number of vertices in a cluster and $\mathrm{N}$ is the number of frames in the animation. Performing singular value decomposition (SVD) for A obtains:

$$
A=B \cdot S \cdot V^{T}
$$

Therein, matrix B and V are both orthogonal matrices, while matrix $S$ is a diagonal matrix. The columns of matrix $\mathrm{B}$ and $\mathrm{V}$ are linearly independent. The values of the diagonals of matrix $\mathrm{S}$ are singular, corresponding to principal components $\mathrm{B}$ and $\mathrm{V}$. The singular values of the diagonals decrease from the upper-left to the lower-right. In other words, PCs closer to the front have larger singular values, expressing more important vertex coordinates and more important key-motions in the original animation. From the perspective of dimension reduction, PCA only stores the first $\rho$ columns of matrices $\mathrm{B}, \mathrm{V}$, and $\mathrm{S}$, using the subspace formed by the first $\rho$ to reconstruct approximated animation. A larger $\rho$ indicates a greater number of reserved principal components (more required storage and memory usage) and more similarity between A' and A (smaller error).

Under a client-server framework, the PCs possessed by the server (the encoding side), must be complete; otherwise, it must be certain that extremely low error can be guaranteed following reconstruction. According to our experiment, when K-mean clustering is performed for an animation with 10 clusters, the server side can provide almost entirely undistorted animation for the first 50 PCs stored by each cluster $(\rho=50)$. In most applications, $\rho=20$ is already sufficient.

\subsection{Progressive Display for Dynamic 3D Meshes}

To reconstruct 3D animation from reduced PCs, matrix multiplications are performed for $\mathrm{PCs} \mathrm{B}, \mathrm{S}$, and V:

$$
A^{\prime}=\sum_{i=1}^{\rho} B_{i} \cdot S_{i i} \cdot V_{i}^{T}
$$

In addition, after studying the linear independence of B, S, and V, we find that after referring to Fig. 2, Formula (2) can be rewritten as:

$$
A^{\prime}=B_{1} \cdot S_{11} \cdot V_{1}^{T}+B_{2} \cdot S_{22} \cdot V_{2}^{T}+\cdots+B_{\rho} \cdot S_{\rho \rho} \cdot V_{\rho}^{T}
$$

Therein, $\mathrm{B}_{1}, \mathrm{~S}_{11}$, and $\mathrm{V}_{1}$ are referred to as the first PC $\mathrm{R}_{1} ; \mathrm{B}_{2}, \mathrm{~S}_{22}$, and $\mathrm{V}_{2}$ are the second $\mathrm{PC} R_{2}$, and so forth. Each PC included two sets of vectors and one set of scalars. The first PC held the most basic information in the process of animation reconstruction, while the other PCs could be viewed as offsets of the first PC. This characteristic allowed reconstruction and transmission iteratively. Collecting the geometric matrix of each cluster $\mathrm{k}$ for each PC allowed us to obtain the base vertex coordinates for the entire animation:

$$
\begin{aligned}
& \left\{\Phi_{1}^{1}, \Phi_{1}^{2}, \ldots, \Phi_{1}^{K}\right\} \\
& \Phi_{1}^{\kappa}=\mathrm{B}_{1}^{\kappa} \cdot \mathrm{S}_{11}^{K} \cdot\left(\mathrm{V}_{1}^{K}\right)^{T}, \forall \kappa=1 \ldots \mathrm{K}
\end{aligned}
$$

With vertex coordinate information and connectivity obtained from the server side, we could reconstruct the animation with the coarsest resolution, referred to as the base animation $\mathrm{A}^{1}$.
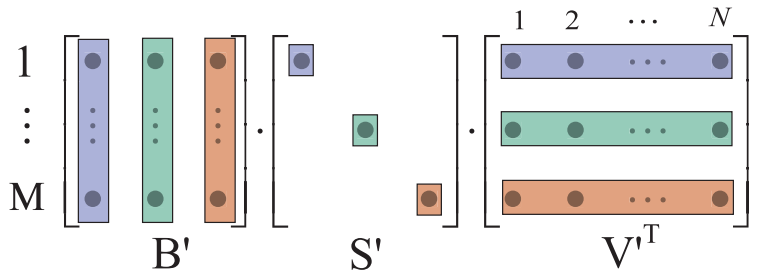

Fig. 2 Different colors in the figure represent different PCs, each of which is linearly independent from the others; each transmission of one color (one PC) allows for one update. 


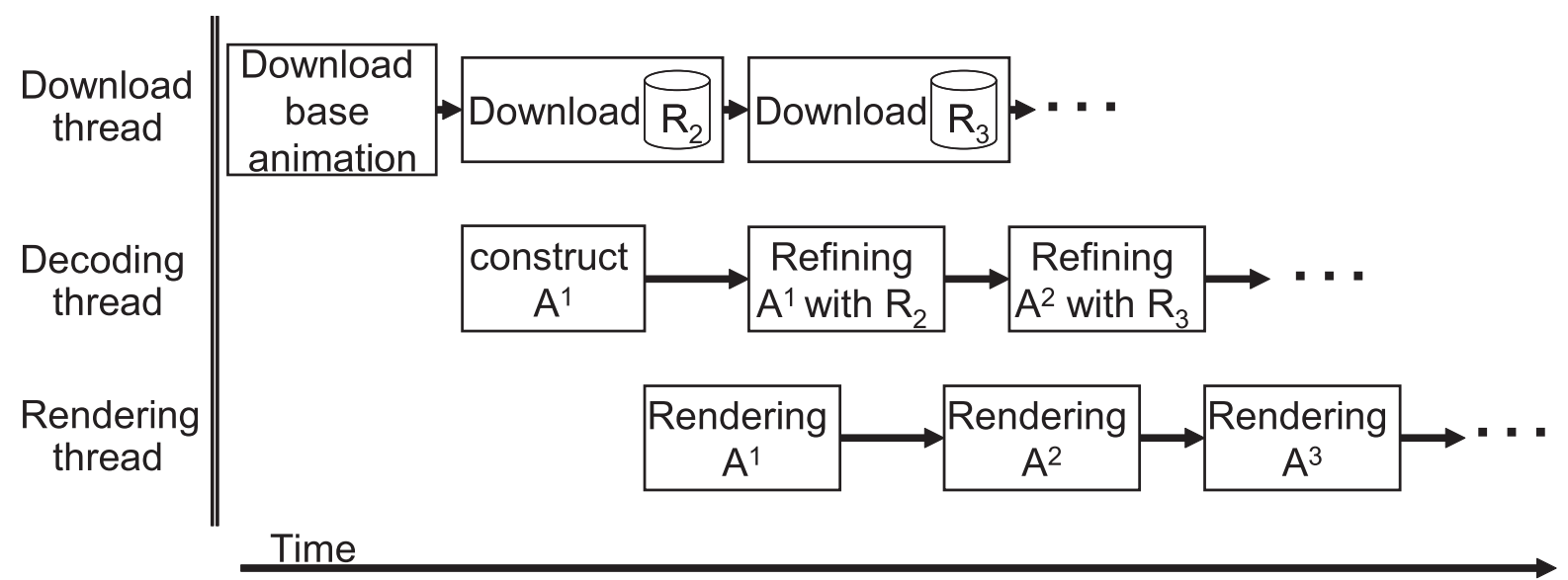

Fig. 3 This diagram illustrates how progressive display functions at the decoding side. Although the client should download, decompress and render simultaneously, the decompression and rendering thread could be processed in real time. Therefore, transmitting the refined operators is the bottleneck which depends on the network speed.

Once the decoding side reconstructed the base animation, the animation $\mathrm{A}^{1}$ could be viewed while receiving PCs to perform the following updates, as shown in Fig. 3. With the continued transmission and update of $\mathrm{PCs} \mathrm{R}_{2}, \mathrm{R}_{3}, \ldots$, the animation viewed by the user becomes increasingly refined $A^{1}, A^{2}, A^{3}, \ldots$ The PCs $R_{2}, R_{3}, \ldots$ used to improve resolution are referred to as refined operators.

Please note that each refined operator contained only the $i^{\text {th }} P C\left(B_{i}, S_{i i}, V_{i}\right)$ for a specific cluster; each refined operator did not include the $\mathrm{i}^{\text {th }} \mathrm{PC}$ for every cluster. This stipulation is one of the major differences between this paper and Sattler's work [5]. Dividing the PCs of each cluster into refined operators reduced the amount of data that each refined operator had to transmit, and at the same time, it increased the frequency of updates. For visualization, the process of updates is more frequent and therefore smoother than collecting PCs of every cluster into one refined operator.

Because the singular value matrix $\mathrm{S}$ had already calculated the importance of all PCs in a cluster, the update sequence of refined operators in that cluster would be singular. Assume the geometric coordinate matrix of a cluster is $\Phi_{1}, \Phi_{2}, \ldots, \Phi_{\rho}$ with different resolutions. Aside from $\Phi_{1}$, which was obtained using the first PC, $\Phi_{i}$ was calculated using $\Phi_{i-1}$ and the refined operator $R_{i}$ :

$$
\begin{aligned}
\Phi_{\mathrm{i}} & =\Phi_{\mathrm{i}-1}+\Delta_{\mathrm{i}} \\
\Delta_{\mathrm{i}} & =\mathrm{B}_{\mathrm{i}} \cdot \mathrm{S}_{\mathrm{ii}} \cdot \mathrm{V}_{\mathrm{i}}^{\mathrm{T}}
\end{aligned}
$$

Having obtained $\Phi_{i}$, the decoding side should receive the $R_{\mathrm{i}}$ in order to calculate $\Delta_{\mathrm{i}}$, and applying formula (5) to refine the resolution of $\Phi_{i-1}$ into $\Phi_{i}$.

Compression methods based on PCA are hard to determine how many PCs $\rho$ would be appropriate. One common method is to have the number of PCs determined by the user, prior to compression [2], [5]. Lee [24] introduced an automatic method that determines $\rho$ by retaining PCs with singular values greater than 0 . In our method, the client could continue updating and improving the resolution of anima- tion as long as the server continued to transmitting refined operators. This idea implies the PCA based method could avoid solving the problem of determining number of PCs. If viewers felt that the resolution was adequate, they could stop receiving and updating to save bandwidth and memory at any time.

\subsection{Determining the Sequence of Refined Operators}

Utilizing Clustered PCA can reduce the required storage and maintain an acceptable level of error [25]. Unfortunately, this produces another problem, "Which refined operator (between different clusters) should be transmitted first?" Assuming that $\mathrm{k}$ clusters exist and each cluster has $\mathrm{r}$ refined operators, there should be $\mathrm{k} \times \mathrm{r}$ refined operators of the animation. For permuting these refined operators, even discounting the order of operators in the same cluster is singular, there would still be $\frac{(k \times r) !}{(r !)^{k}}$ possibilities. As it is almost impossible to obtain the optimal sequence through brute force, an approximate solution must be sought.

Fortunately, transmitting the PCs of each cluster based on cluster sequence is a reasonable approximate solution, as shown the following sequence:

$$
\mathrm{R}_{1}^{1}, \mathrm{R}_{1}^{2}, \ldots, \mathrm{R}_{1}^{\mathrm{K}}, \mathrm{R}_{2}^{2}, \ldots, \mathrm{R}_{2}^{\mathrm{K}}, \ldots, \mathrm{R}_{\rho}^{1}, \ldots, \mathrm{R}_{\rho}^{\mathrm{K}-1}, \mathrm{R}_{\rho}^{\mathrm{K}}
$$

This type of progressive transmission method is referred to as round-robin progressive transmitting (RPT).

RPT provides a simple and fast solution that achieves progressive transmitting, and does not require the user to select the number of PCs. A low error and required data for progressive animation can be achieved by transmitting the interleaved refined operators. However, we discovered significant correlation between singular values and reconstructed error through after investigating the characteristics of the PCs. The higher the singular value of a PC was, the greater the error could be lowered, but the size of the singular value differed according to the distribution of vertex 
coordinates (or the bounding box) and factors such as the number of vertices and the degree of change. Therefore, the size of the singular value could not be used to measure the importance between PCs of different clusters directly, as the basis for measurement. Instead, a normalized method had to be used first, as in the following formula:

$$
C_{i}^{k}=\frac{\sigma_{i}^{k}}{\sum_{j} \sigma_{j}^{k}}
$$

$C_{i}^{k}$ represents the percentage of variance of the $i^{\text {th }}$ component in the $\mathrm{k}^{\text {th }}$ cluster and illustrates the importance of this component in the $\mathrm{k}^{\text {th }}$ cluster.

After $C_{i}^{k}$ was obtained, the variance between different clusters should also be considered, as the effects of clusters with greater numbers of vertices have more significant effects on the entire animation. Thus, we increased the priority order of PCs belonging to clusters with greater numbers of vertices in anticipation of further decreasing error. The $\mathrm{W}_{\mathrm{i}}^{\mathrm{k}}$ of refined operator measurement after adjustment is as follows:

$$
W_{i}^{k}=\left(\frac{M_{k}+N}{M+N}\right) \cdot C_{i}^{k}
$$

In this formula, $\mathrm{M}_{\mathrm{k}}$ represents the number of vertices in the $\mathrm{k}^{\text {th }}$ cluster, while $\mathrm{M}, \mathrm{N}$ represent the total number of vertices and frames in the entire animation, respectively. The order of transmission of refined operators was determined by the value of $\mathrm{W}_{\mathrm{i}}^{\mathrm{k}}$. The larger the $\mathrm{W}_{\mathrm{i}}^{\mathrm{k}}$ of a $\mathrm{PC}$ is, the higher its transmission priority in the decompression and the earlier it would be used for reconstruction and updating. Finally, we refer the order of progressive transmission obtained from the number of vertices, number of frames, and percentage of variance, as weighted progressive transmitting (WPT).

In recording the transmission order of WPT, simply recording cluster number $\mathrm{k}$ based on the value of $\mathrm{W}_{i}^{\mathrm{k}}$ was acceptable, as there was only one transmission order for PCs in the same cluster. For example, if $\mathrm{W}_{1}^{1}>\mathrm{W}_{1}^{2}>$ $\mathrm{W}_{2}^{2}>\mathrm{W}_{3}^{2}>\mathrm{W}_{2}^{1}$, only $1,2,2,2,1$ would have to be recorded. Once the decoding side received this order, it would be able to interpret the following refined operator order as $\mathrm{R}_{1}^{1}>\mathrm{R}_{1}^{2}>\mathrm{R}_{2}^{2}>\mathrm{R}_{3}^{2}>\mathrm{R}_{2}^{1}$.

For discussing the WPT and RPT in detail, the difference in the compression stage would be the requirement of WPT to calculate the $\mathrm{W}_{\mathrm{i}}^{\mathrm{k}}$ for each PC, while the order used by RPT would not require special calculation. During the decompression stage, WPT would require the identification of the following PC with its cluster, and would therefore require additional overhead for transmitting the order of operators, while the RPT could waive the transmission overhead for this portion. However, we demonstrated that the additional overhead of WPT was extremely low. Nonetheless, we were able to reconstruct 3D animation more accurately than RPT could, without non-continuous boundary surfaces. This is illustrated in the results section.

\section{Results}

The experiment outlined in this study was performed on a computer with an Intel Core 2 Q6600 $(2.4 \mathrm{GHz})$ and $2 \mathrm{~GB}$ of RAM. Table 1 lists the time required to execute each encoding stage, where the number of clusters $\mathrm{K}=10$ in this table. From this data it can be seen that although the majority of time was spent on clustering, this only needed to be performed once for the K-mean and SVD of an animation (once for each cluster for SVD). The following PCs could be then reused. Table 1 also shows that the rapid calculation speed of WPT order was sufficient to support real-time applications. The size of the WPT sequence represents the additional storage required to record/transmit the order of PCs in the WPT method. None of the models exceeded $3 \mathrm{~KB}$, and therefore had a negligible effect on compression.

Figure 4 lists the processes of our system displaying animation progressively. Here we should particular explain the figures in the left-most column (which is the coarsest version). Although it appears that this version has a number of flaws (non-continuous surface and distortion), it is only a single frame sampled from the progressive animation playback process. As can be seen from the data size, with continual updating, higher quality animation could be seen within seconds. Furthermore, the required data size for this type of high quality animation was only $400 \mathrm{~KB}$.

Figure 4 also shows clear non-continuity in the boundaries between clusters in RPT, while WPT does not display this condition due to the variance between the clusters considered. Table 2 shows the RMSE (root mean squared error) of each cluster after compression with 6 clusters $(K=6)$. We observed a significant difference between the RMSE of each cluster when using RPT reconstruction, while the RMSE of WPT reconstruction were relative close. Thus, the disparity in quality after reconstruction was believed to be the main cause behind non-continuity in the boundaries between clusters. Due to insufficiencies in the number of PCs in certain clusters, the distortion in these clusters was more severe, affecting the presentation of the entire animation. The more visualized results of WPT are listed in Fig. 5.

In comparing reconstruction error, although a common standard to measure the quality of reconstructed animation does not yet exist, several tools could be used to achieve this. In addition to the previously used RMSE, KG error [7] would be another. KG error is a concept of relative error; if the variance in an animation is more serious, the higher degree of error would be allowed. This is different from

Table 1 Basic data for animation testing and execution time for compression in seconds. The number of clusters $\mathrm{K}=10$ in this table.

\begin{tabular}{c||cc||ccc||c}
\hline \multicolumn{1}{c||}{ Model } & Vertex & Frame & \multicolumn{3}{|c||}{ Encoding time } & Size of \\
& & & K-mean & SVD & WPT & WPT seq. \\
\hline Chicken & 3030 & 400 & 25.03 & 14.66 & 0.0418 & $1.5 \mathrm{~KB}$ \\
Cowheavy & 2904 & 204 & 10.88 & 5.23 & 0.0416 & $1.4 \mathrm{~KB}$ \\
Dance & 7061 & 201 & 26.98 & 25.64 & 0.0499 & $2.7 \mathrm{~KB}$ \\
Horse & 8431 & 48 & 4.484 & 0.57 & 0.0379 & $0.7 \mathrm{~KB}$ \\
\hline
\end{tabular}



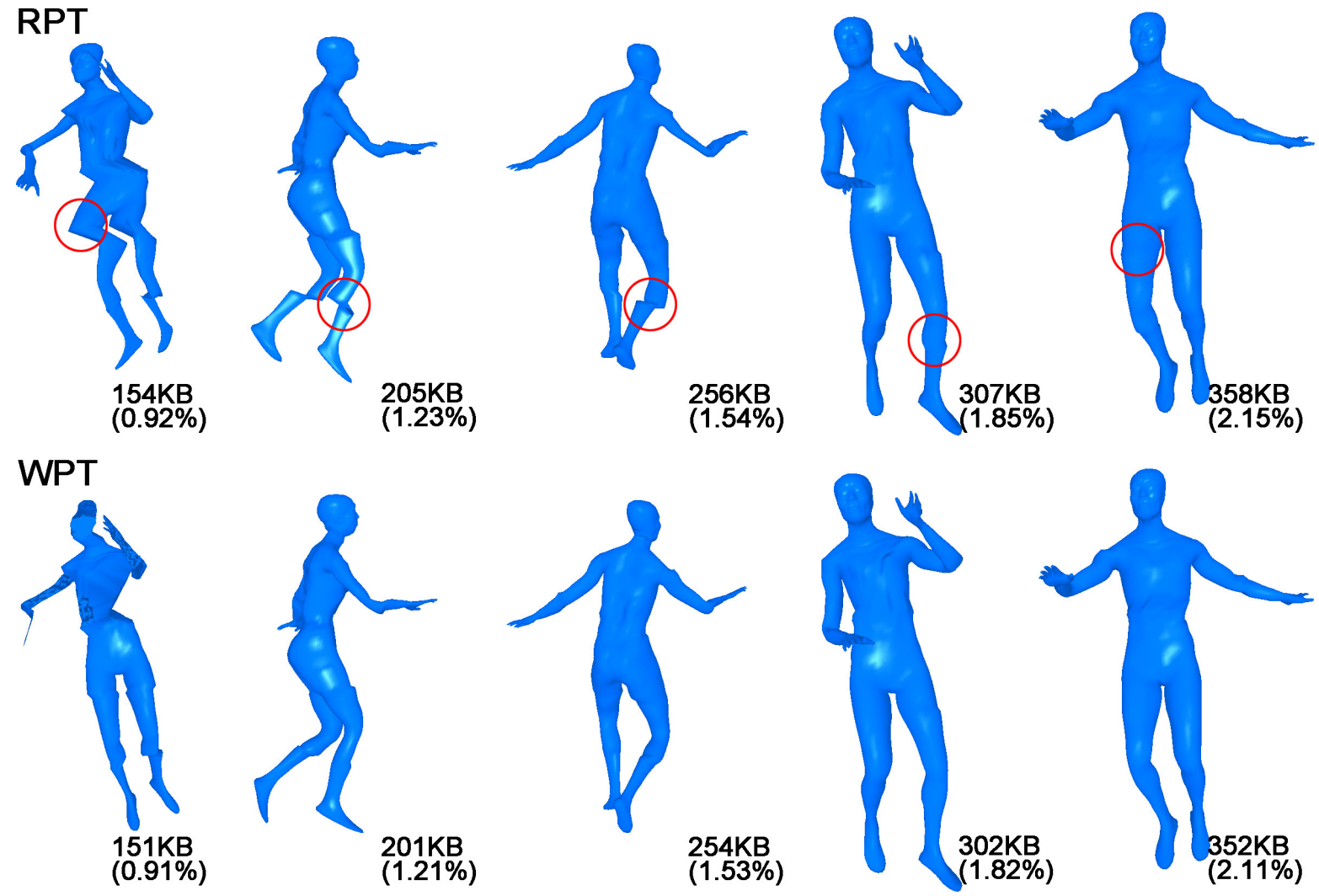

\section{Original (16MB)}
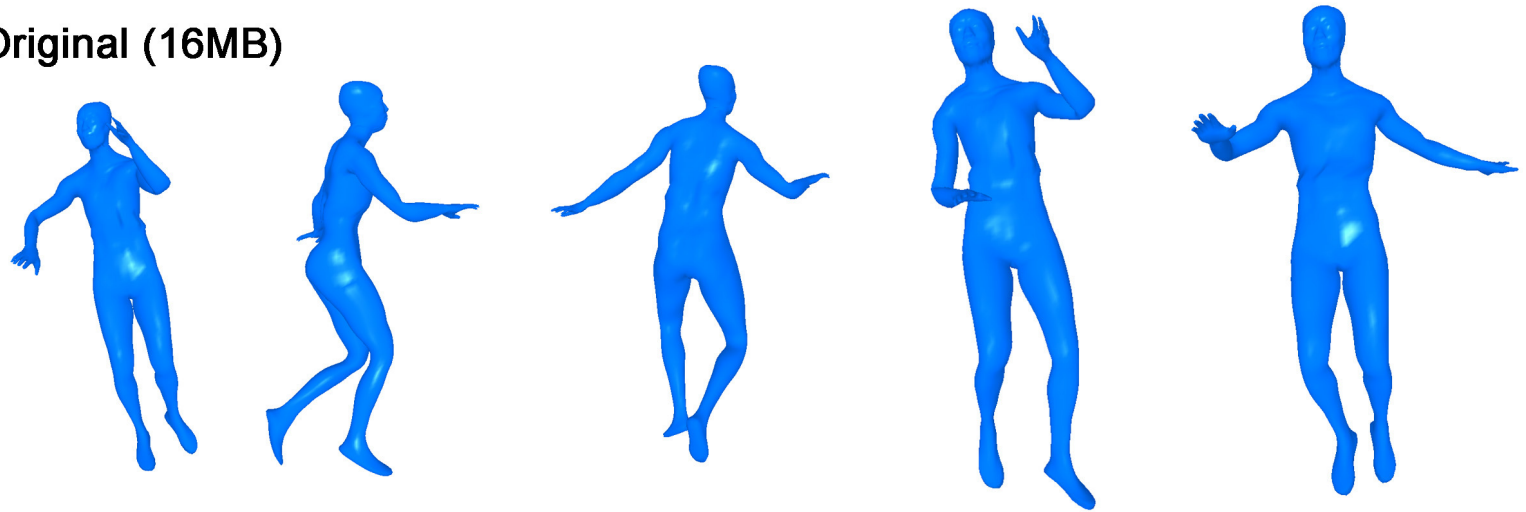

Fig. 4 Comparison of dance animation utilizing RPT and WPT updating procedures. The red circles show the portions of clear boundary non-continuity in RPT, while the effect displayed by WPT was distinctly superior. The WPT presents reasonable visual results by using no more than $3 \%$ original size.

Table 2 There are 6 clusters in the dance animation. The RMSE $\left(\times 10^{-5}\right)$ of each cluster was compared using RPT and WPT transmitting similar amounts of data.

\begin{tabular}{c|c|cccccc}
\hline \multicolumn{2}{c|}{ Cluster no. } & 1 & 2 & 3 & 4 & 5 & 6 \\
\hline \multirow{2}{*}{ RPT } & $\rho$ & 15 & 15 & 15 & 15 & 15 & 15 \\
& RMSE & 0.06 & 2.84 & 0.03 & 0.03 & 8.52 & 4.56 \\
\hline \multirow{2}{*}{ WPT } & $\rho$ & 14 & 17 & 11 & 10 & 19 & 17 \\
& RMSE & 0.13 & 1.55 & 0.07 & 0.74 & 3.29 & 0.75 \\
\hline
\end{tabular}

RMSE, in which the error value would increase when measuring animations with a greater degree of variance. Figure 6 uses $\mathrm{KG}$ error to compare our method with previous methods. The order of WPT updating obtained through optimal refined operators showed a significant advantage over Sattler's method, even though our compression method was no more careful during the clustering stage. This also demonstrated that the K-mean clustering was sufficient to handle 3D animation compression. The PC reconstruction order was more important than optimization during the clustering stage.

Other than KG error, Lee [17] applied peak signal to noise ratio (PSNR), a tool commonly used to measure error in $2 \mathrm{D}$ images, to $3 \mathrm{D}$ space to observe the interference relationship between two 3D static models. The higher the PSNR was, the more similar the two models were; the lower the interference, the lower the error. Using PSNR to measure $3 \mathrm{D}$ animation required that each frame in the anima- 


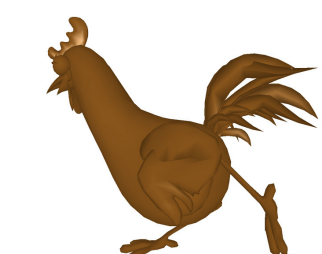

$548 K B$

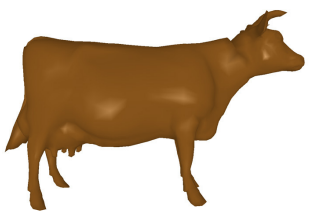

$154 K B$

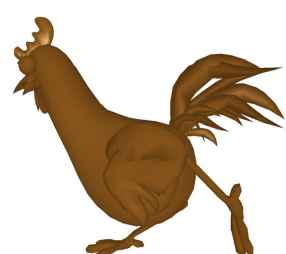

Original

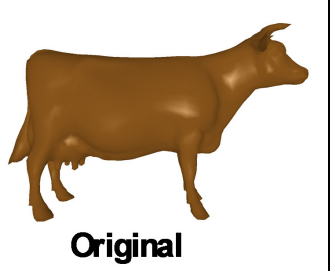

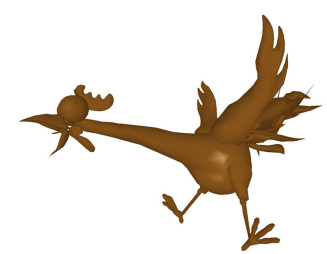

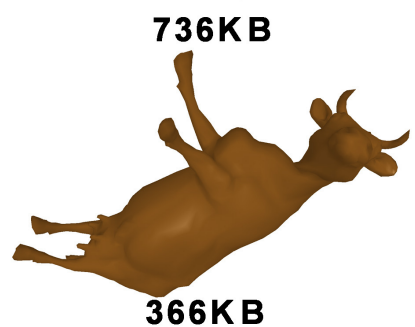

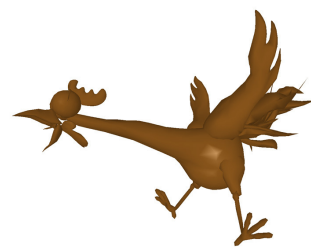

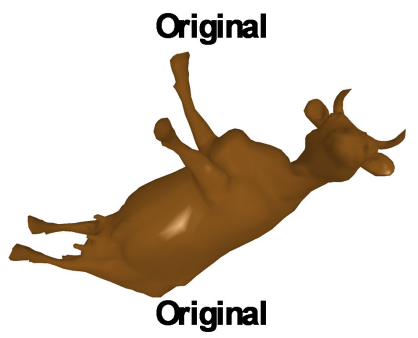

Fig. 5 Sample frames of chicken and cowheavy animation reconstructed by WPT. Comparing to original frames, WPT presents well visualization at limited storage.
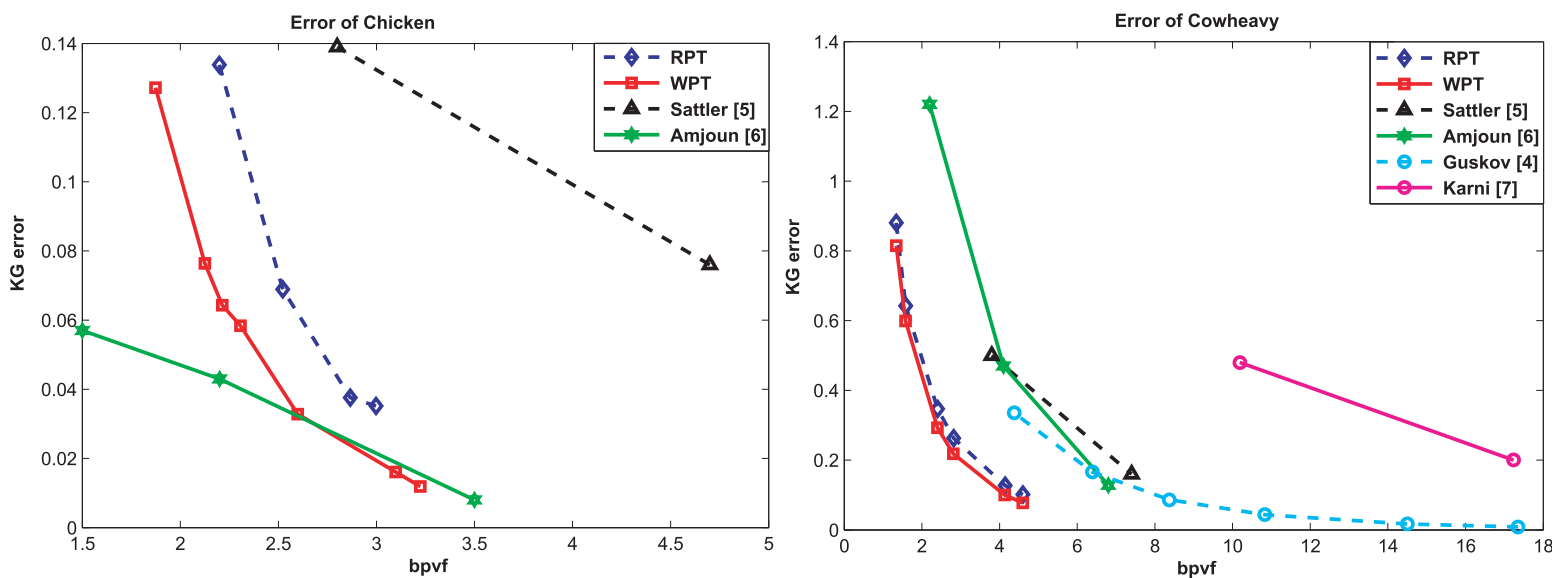

Fig. 6 Using KG error [7] to compare this study with other previous compression methods [4]-[7]. The figures show that the method used in this study had a reasonable compression rate, with a reasonable degree of error.

Table 3 Compare RPT and keyframe based methods by using PSNR. The keyframe based method data is referenced from [17].

\begin{tabular}{|c|c|c|c|c|c|c|c|}
\hline & \multirow{2}{*}{\multicolumn{3}{|c|}{$\frac{\text { PCA based method }}{\text { RPT }}$}} & \multicolumn{4}{|c|}{ Keyframe based method } \\
\hline & & & & & & Huang [16] & Lee [17] \\
\hline & $\rho$ & Data size $(\mathrm{KB})$ & Avg. PSNR & \# of keyframe & Data size $(\mathrm{KB})$ & Avg. PSNR & Avg. PSNR \\
\hline \multirow{3}{*}{ Chicken } & 10 & 602 & 57.40 & 20 & 758 & 47.63 & 46.67 \\
\hline & 20 & 1200 & 81.82 & 40 & 1512 & 60.14 & 59.35 \\
\hline & 30 & 1800 & 95.57 & 50 & 1888 & 66.24 & 64.75 \\
\hline \multirow{3}{*}{ Dance } & 10 & 524 & 66.26 & 10 & 855 & 33.87 & 33.23 \\
\hline & 30 & 1570 & 119.49 & 20 & 1709 & 44.36 & 43.41 \\
\hline & 50 & 2620 & 119.99 & 50 & 4267 & 62.83 & 62.14 \\
\hline
\end{tabular}

tion be considered a static model. The corresponding initial models for each static model would compare with PSNR. The average value of PSNR (Avg. PSNR) was then determined to decide the quality of the reconstructed animation. Due to PCA based method is different from keyframe based method [16], [17], we should compare the average PSNR with similar data size. Table 3 shows that even RPT could reconstruct animation with lower distortion than keyframe based method.

\section{Conclusion and Future Work}

On-line multimedia data applications have become increasingly sophisticated, prompting internet users to value mobile access to the internet. This study proposed a progressive representation of dynamic 3D meshes, which not only compresses the data storage requirements, but also presents the animation in a new way. With the framework proposed in this study, 3D animation could offer multiple resolutions, 
streamed across the internet. The method of expression would be similar to that of the JPEG progressive mode, capable of gradual enhancement from coarse animation to more refined. The framework proposed in this study begins by compressing 3D animations using K-mean clustering with PCA, with the linearly independent characteristic of each PC performing updates independently. In this manner, each PC could become both the unit for streaming, as well as the refined operator for updating animation.

Clustered PCA increases the number of refined operators, as well as the complexity in their selection. This study proposed using the RPT and WPT methods to decide the transmitting order. RPT method transferred the average of each cluster and did not require the recording of any data, and WPT analyzed the weight of each PC. The PCs belonging to clusters with greater influence had greater weight, and higher priority to transmit/update. Although the sequence of WPT was transmitted to the decoding side resulting in additional transmission overhead, we have shown that that WPT produces significantly lower error compared to RPT, under conditions with similar data size. Furthermore, WPT balanced the error of each cluster, thus largely overcoming the problem of non-continuity boundary because the resolution of each cluster becomes more similar.

When the decoding side displayed progressive animation, viewers could only see coarse and blurred animations due to the low data size that was initially received. With continual animation updating, viewers could clearly see an increase in the clarity of the animation. Furthermore, the structure of the data after compression makes more efficient use of bandwidth. The total transmission data was far lower than that of traditional 3D animation display methods over the course of viewing animation.

In future work, we hope to further simplify and compress connectivity, in addition to considering the geometric data of 3D animation. For example, with applying edge-driven [19] or valence-driven [20] compression methods could express changes in space (mesh) and time (geometric) progressively. With changes in $2 \mathrm{D}$ (space and time), the relationship between different dimensions had to be carefully considered. When the mesh was simplified, the total number of vertices in the entire animation should be reduced. The PCs compressed in this study had to be used in coordination with simplified mesh. Furthermore, through the inspiration obtained in the basic keyframe method [16], [17] and the concept that PCs could be further compressed and predicted [9], we believe that PCs could be further configured to frames. For example, conversion methods for extracting key frames to extracting key PCs so that only key PCs would need to be stored. Other PCs could be calculated by combining key PCs with prediction methods. Finally, finding a superior compression method and a visually superior progressive display method is one goal for us to pursue in the future.

\section{References}

[1] J.E. Lengyel, "Compression of time-dependent geometry," Proc. ACM Symposium on Interactive 3D Graphics, pp.89-95, 1999.

[2] M. Alexa and W. Müller, "Representing animations by principal components," Computer Graphics Forum, vol.19, no.3, pp.411-418, 2000.

[3] H.M. Briceño, P.V. Sander, L. McMillan, S. Gortler, and H. Hoppe, "Geometry videos: A new representation for 3D animations," Proc. ACM SIGGRAPH/Eurographics Symposium on Computer Animation, pp.136-146, 2003.

[4] I. Guskov and A. Khodakovsky, "Wavelet compression of parametrically coherent mesh sequences," Proc. ACM SIGGRAPH/Eurographics Symposium on Computer Animation, pp.183-192, 2004.

[5] M. Sattler, R. Sarlette, and R. Klein, "Simple and efficient compression of animation sequences," Proc. ACM SIGGRAPH/Eurographics Symposium on Computer Animation, pp.209-217, 2005.

[6] R. Amjoun and W. Straßer, "Efficient compression of 3D dynamic mesh sequences," J. WSCG, vol.15, no.1-3, pp.99-106, 2007.

[7] Z. Karni and C. Gotsman, "Compression of soft-body animation sequences," Comput. \& Graph., vol.28, no.1, pp.25-34, 2004.

[8] L. Vasa and V. Skala, "Coddyac: Connectivity driven dynamic mesh compression," 3DTV Conference, 2007, pp.1-4, 2007.

[9] L. Vasa and V. Skala, "Combined compression and simplification of dynamic 3D meshes," Computer Animation and Virtual Worlds, vol.20, no.4, pp.447-456, 2009.

[10] T.Y. Lee, Y.S. Wang, and T.G. Chen, "Segmenting a deforming mesh into near-rigid components," The Visual Computer, vol.22, no.9, pp.729-739, 2006.

[11] K. Mamou, T. Zaharia, and F. Prêteux, "A skinning approach for dynamic 3D mesh compression," Computer Animation and Virtual Worlds, vol.17, no.3-4, pp.337-346, 2006.

[12] G. Collins and A. Hilton, "A rigid transform basis for animation compression and level of detail," Vision, Video, and Graphics, pp.17, 2005.

[13] D.L. James and C.D. Twigg, "Skinning mesh animations," ACM Trans. Graph., vol.24, no.3, pp.399-407, 2005.

[14] L. Ibarria and J. Rossignac, "Dynapack: Space-time compression of the 3D animations of triangle meshes with fixed connectivity," Proc. ACM SIGGRAPH/Eurographics Symposium on Computer Animation, pp.126-135, 2003.

[15] X. Gu, S.J. Gortler, and H. Hoppe, "Geometry images," ACM Trans. Graph., vol.21, no.3, pp.355-361, 2002.

[16] K.S. Huang, C.F. Chang, Y.Y. Hsu, and S.N. Yang, "Key probe: A technique for animation keyframe extraction," The Visual Computer, vol.21, no.8, pp.532-541, 2005.

[17] T.Y. Lee, C.H. Lin, Y.S. Wang, and T.G. Chen, "Animation keyframe extraction and simplification using deformation analysis," IEEE Trans. Circuits Syst. Video Technol., vol.18, no.4, pp.478486, 2008.

[18] L. Vasa and V. Skala, "Cobra: Compression of the basis for PCA represented animations," Computer Graphics Forum, vol.28, no.6, pp.1529-1540, 2009.

[19] J. Rossignac, "Edgebreaker: Connectivity compression for triangle meshes," IEEE Trans. Vis. Comput. Graphics, vol.5, no.1, pp.47-61, 1999.

[20] P. Alliez and M. Desbrun, "Progressive compression for lossless transmission of triangle meshes," Proc. ACM SIGGRAPH 2001, pp.198-205, 2001.

[21] G. Liu and L. McMillan, "Segment-based human motion compression," Proc. ACM SIGGRAPH/Eurographics Symposium on Computer Animation, Eurographics Association, Vienna, Austria, 2006.

[22] O. Arikan, "Compression of motion capture databases," ACM Trans. Graph., vol.25, no.3, pp.890-897, 2006. 
[23] T. Kanungo, D.M. Mount, N.S. Netanyahu, C.D. Piatko, R. Silverman, and A.Y. Wu, "A local search approximation algorithm for k-means clustering," Computational Geometry: Theory and Applications, vol.28, pp.89-112, 2004.

[24] P.F. Lee, C.K. Kao, J.L. Tseng, B.S. Jong, and T.W. Lin, “3D animation compression using affine transformation matrix and principal component analysis," IEICE Trans. Inf. \& Syst., vol.E90-D, no.7, pp.1073-1084, July 2007.

[25] K. Mamou, T. Zaharia, and F. Prêteux, "A preliminary evaluation of 3D mesh animation coding techniques," Proc. SPIE Conference on Methematical Methods in Pattern and Image Analysis, pp.44-55, 2005.

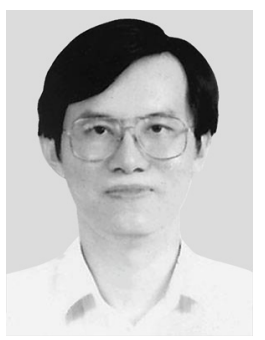

Bin-Shyan Jong received the B.S. degree in computer science from Chung Yuan Christian University in 1978, and the M.S. and Ph.D. degrees from the Institute of Computer Science at National Tsing Hua University in 1983 and 1989. He is a professor in the Dept. of Information and Computer Engineering, Chung Yuan Christian University. His research interests include computer graphics and computer aided education.

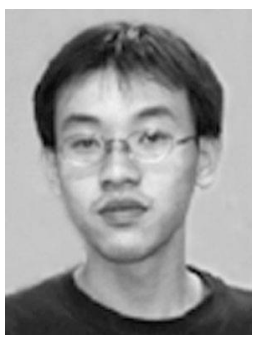

Chi-Kang Kao received the B.S. and M.S. degrees in computer engineering from Chung Yuan Christian University, Taiwan. He is now a Ph.D. candidate in electronic engineering at Chung Yuan Christian University, Taiwan. His research interests include computer graphics and computer animation.

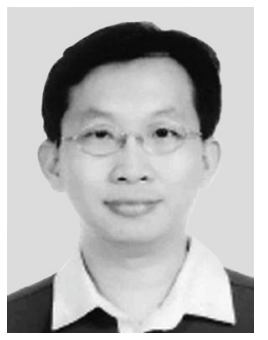

Juin-Ling Tseng received his B.S. from SooChow University in 1994 and M.S./Ph.D. from Chung Yuan Christian University, Taiwan, in 1996/2006. Currently, he is an assistant professor in the Dept. of Computer Science and Information Engineering at the Minghsin University of Science and Technology, Taiwan. His research interests include the areas of computer graphics.

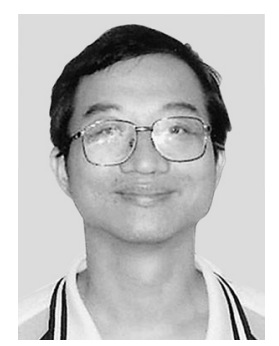

Tsong-Wuu Lin received the B.S. degree in Computer Science from Tamkang University in 1985. He received the M.S. and Ph.D. degree in Computer Science in 1987 and 1991 at National Tsing Hua University, Hsinchu, Taiwan. He is now a professor in Dept. of Computer and Information Science, Soochow University. His research interests include computer graphics and computer aided education. 